\title{
Dynamical Analysis of a Mathematical Model of COVID-19 Spreading on Networks
}

\author{
Wang $\mathrm{Li}^{1}$, Xinjie $\mathrm{Fu}^{2}$, Yongzheng Sun ${ }^{1}$ and Maoxing $\mathrm{Liu}^{2 *}$ \\ ${ }^{1}$ School of Mathematics, China University of Mining and Technology, Xuzhou, China, ${ }^{2}$ School of Science, North University of \\ China, Taiyuan, China
}

In this article, an SEAIRS model of COVID-19 epidemic on networks is established and analyzed. Following the method of the next-generation matrix, we derive the basic reproduction number $R_{0}$, and it shows that the asymptomatic infector plays an important role in disease spreading. We analytically show that the disease-free equilibrium $E_{0}$ is asymptotically stable if $R_{0} \leq 1$; moreover, the effects of various quarantine strategies are investigated and compared by numerical simulations. The results obtained are informative for us to further understand the asymptomatic infector in COVID-19 propagation and get some effective strategies to control the disease.

Keywords: COVID-19 epidemic, networks, asymptomatic, the basic reproduction number, quarantine

\section{OPEN ACCESS}

Edited by:

Chengyi Xia,

Tianjin University of Technology, China

Reviewed by:

Chuansheng Shen,

Anqing Normal University, China

Weigang Sun,

Hangzhou Dianzi University, China

*Correspondence:

Maoxing Liu

liumaoxing@126.com

Specialty section:

This article was submitted to

Social Physics,

a section of the journal

Frontiers in Physics

Received: 01 September 2020

Accepted: 25 November 2020

Published: 18 January 2021

Citation:

Li W, Fu X, Sun Y and Liu M (2021)

Dynamical Analysis of a Mathematical

Model of COVID-19 Spreading

on Networks.

Front. Phys. 8:601459.

doi: 10.3389/fphy.2020.601459

\section{INTRODUCTION}

Coronaviruses are a group of enveloped viruses with a positive-sense, single-stranded RNA and viral particles resembling a crown from which the name derives. They belong to the order of Nidovirales, family of Coronaviridae, and subfamily of Orthocoronavirinae. A recent coronavirus outbreak has started since December 2019. So far, the new virus has infected more than 25 million people and killed at least 842,000 of them [1-3].

In order to better study the spread and control of infectious diseases, several classical mathematical models have been discussed to understand the transmission mechanism and dynamics of the disease, such as the SI model, SIR model, SEIR model, and SEIRS model [4, 5]. Different factors are considered in different models, and the purpose of these models is finding the transmission mechanism and dynamics of the diseases for controlling the diseases. This process needs a contact between them. It is found that the best pattern of contact is contact in the networks [6-11], and study epidemic model on networks has been a hot field in mathematical biology $[6-20,35]$.

To find the transmission mechanism, we have to know who is the infector [27]. An infector is an individual who carries the virus and can export the virus. Sometimes, an individual with the infected state may not show any symptoms of disease, and the state can be defined as asymptomatically infected. An individual with the infected state who shows symptoms of disease can be defined as symptomatically infected. Exposed state and asymptomatically infected state are more dangerous than symptomatically infected state. When a susceptible individual contacts a symptomatic one, he(she) will usually do something possible to prevent the disease spreading.

In the disease spreading process, a symptomatic infector will be restricted by individual behavior or public behavior, but a symptomatic one has a higher efficiency than an asymptomatic one. A class of works studied the role of symptomatic infectors and 
asymptomatic infectors in diseases infections, such as dengue virus [21, 22], malaria [23], and Norovirus [24-27]. The results of these works all show that the asymptomatic cases cannot be neglected. Recently, several mathematic models within asymptomatic infection have been studied, such as $S A I R$ model [21], CI model [28], SEIADR model [29], and $S E I I_{e} Q R$ model [30]. But mathematical models within asymptomatic infection on networks are still relatively rare. When the COVID-19 pandemic begin to outbreak, quarantining certain individuals may be the most efficient way to stop the outbreak of disease, but the choice of which is an important step in the control process, and thus we also discuss the effect of different quarantine strategies.

In this article, we consider an SEAIRS epidemic model on scale-free networks to study the spread of COVID-19. Further, we consider the effect of the asymptomatic state changing into the symptomatic state. By studying the dynamics of the model, we try to find what role the three infectors, $E, A$, and $I$, play in disease spreading, especially for the asymptomatic infectors, $E$ and $A$. Next, in Section 2, we establish an SEAIRS COVID-19 model on network. In Section 3, we study the dynamics of the model. In Section 4, we study and compare the effects of various control strategies, and carry out numerical simulations to illustrate the theoretical results in Section 5. Finally, conclusions and some discussions are given in Section 6.

\section{THE MODEL}

In the spread process, each node has five states: susceptible $(S)$; exposed $(E)$; asymptomatically infected $(A)$, those who are infectious but have no symptoms; symptomatically infected $(I)$, those who are infectious but have symptoms; and removed $(R)$. The disease transmission flow is depicted in Figure 1. A susceptible individual can be infected by contact at rate $\beta_{1}$ if there are infected individuals with $A$ in its neighbors, or be infected by contact at rate $\beta_{2}$ if there are symptomatically infected individuals $I$. The exposed individual will become $A$ and $I$ with rate $p \alpha$ and $(1-p) \alpha$, and $A$ will become $I$ individual at rate $q$. The infected $A$ and $I$ will become a recovered individual at rate $\mu_{1}$ and $\mu_{2}$ due to treatment, respectively. A recovered individual will become $S$ at rate $\delta$. All parameters are nonnegative.

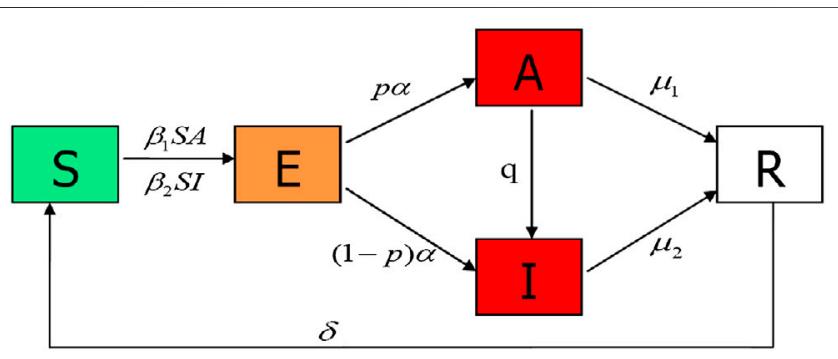

FIGURE 1 | State-transition rules of the SEAIRS model of COVID-19.
In this article, we consider an SEAIRS epidemic model on scale-free networks as follows:

$$
\left\{\begin{array}{l}
\frac{d S_{k}(t)}{d t}=-\beta_{1} k S_{k}(t) \theta_{1}(t)-\beta_{2} k S_{k}(t) \theta_{2}(t)+\delta R_{k}(t), \\
\frac{d E_{k}(t)}{d t}=\beta_{1} k S_{k}(t) \theta_{1}(t)+\beta_{2} k S_{k}(t) \theta_{2}(t)-\alpha E_{k}(t), \\
\frac{d A_{k}(t)}{d t}=p \alpha E_{k}(t)-q A_{k}(t)-\mu_{1} A_{k}(t), \\
\frac{d I_{k}(t)}{d t}=(1-p) \alpha E_{k}(t)+q A_{k}(t)-\mu_{2} I_{k}(t), \\
\frac{d R_{k}(t)}{d t}=\mu_{1} A_{k}(t)+\mu_{2} I_{k}(t)-\delta R_{k}(t) .
\end{array}\right.
$$

Following [6], $\langle k\rangle=\sum_{k} k P_{k}$ describes the average degree and $P_{k}(k=1,2, \ldots, n)$ describes the degree distribution; the probability $\theta_{i}(i=1,2,3)$ is a link pointing to an exposed individual, asymptomatic infected individual, and symptomatic infected individual. Here, $N_{k}$ is a constant that stands for the number of nodes with degree $k$. Then, $N_{k}=S_{k}(t)+E_{k}(t)+$ $A_{k}(t)+I_{k}(t)+R_{k}(t),(k=1,2, \ldots, n)$ and $\sum_{k} N_{k}=N$. We have

$$
\begin{aligned}
& \theta_{1}(t)=\frac{1}{\langle k\rangle} \sum_{k} k P_{k} \frac{A_{k}(t)}{N_{k}}, \\
& \theta_{2}(t)=\frac{1}{\langle k\rangle} \sum_{k} k P_{k} \frac{I_{k}(t)}{N_{k}} .
\end{aligned}
$$

For the practice, the initial condition for model (2.1) satisfies the following:

$\left\{\begin{array}{l}0 \leq S_{k}(0), E_{k}(0), A_{k}(0), I_{k}(0), R_{k}(0) \leq N_{k}, \\ S_{k}(0)+E_{k}(0)+A_{k}(0)+I_{k}(0)+R_{k}(0)=N_{k},\end{array} \quad(k=1,2, \ldots, n)\right.$.

\section{DYNAMICAL BEHAVIORS OF THE MODEL}

In this section, we study the stability of disease-free equilibrium of model (2.1). First, we derive the basic reproduction number of the model.

\subsection{Equilibria and Basic Reproduction Number}

Lemma 3.1. Suppose that $S_{k}(t), E_{k}(t), A_{k}(t), I_{k}(t), R_{k}(t)$ is a solution of model (2.1) satisfying initial conditions of Eq. (2.4), then

$$
\Omega=\left\{\left(S_{k}, E_{k}, A_{k}, I_{k}, R_{k}\right) \mid 0 \leq S_{k}, E_{k}, A_{k}, I_{k}, R_{k} \leq N_{k}, k=1,2, \ldots, n\right\}
$$

is a positive invariant for model (2.1).

The proof of this lemma is similar to that in [31]. We ignore it here. The stability analysis and numerical simulations are investigated in the positive invariant $\Omega$.

It is easy to find that model (2.1) has a disease-free equilibrium $E_{0}$, it is the only susceptible state. $E_{0}=\left(S_{1}^{0}, 0,0,0,0, S_{2}^{0}, 0,0,0,0, \cdots, S_{n}^{0}, 0,0,0,0\right)$, where $S_{k}^{0}=N_{k}$, $k=1,2, \ldots, n$. Following the method of the next-generation matrix [32], the basic reproduction number can be calculated 
by $R_{0}=\rho\left(F V^{-1}\right)$. Here, $F$ is the matrix of the rate of generation of new infections, and $V$ is the matrix of the rate of transfer of individuals out of the two compartments. $F$ is given by

$$
F=\left(\begin{array}{cccc}
A_{11} & A_{12} & \cdots & A_{1 n} \\
A_{21} & A_{22} & \cdots & A_{2 n} \\
\vdots & \vdots & \ddots & \vdots \\
A_{n 1} & A_{n 2} & \cdots & A_{n n}
\end{array}\right)_{3 n \times 3 n}
$$

and

$$
A_{i j}=\left(\begin{array}{ccc}
0 & \frac{\beta_{1} S_{i}^{0} P_{j}}{\langle k\rangle N_{j}} i j & \frac{\beta_{2} S_{i}^{0} P_{j}}{\langle k\rangle N_{j}} i j \\
0 & 0 & 0 \\
0 & 0 & 0
\end{array}\right) .
$$

In addition, $V=\operatorname{diag}\left(B_{1}, B_{2}, \ldots, B_{n}\right)$, where

$$
B_{k}=\left(\begin{array}{ccc}
\alpha & 0 & 0 \\
-p \alpha & q+\mu_{1} & 0 \\
-(1-p) \alpha & -q & \mu_{2}
\end{array}\right) \text {, }
$$

and

$$
B_{k}^{-1}=\left(\begin{array}{ccc}
\frac{1}{\alpha} & 0 & 0 \\
\frac{p}{q+\mu_{1}} & \frac{1}{q+\mu_{1}} & 0 \\
\frac{q+(1-p) \mu_{1}}{\mu_{2}\left(q+\mu_{1}\right)} & \frac{q}{\mu_{2}\left(q+\mu_{1}\right)} & \frac{1}{\mu_{2}}
\end{array}\right) \text {, }
$$

so, $V^{-1}=\operatorname{diag}\left(B_{1}^{-1}, B_{2}^{-1}, \ldots, B_{n}^{-1}\right)$, and the basic reproduction number $R_{0}$ can be obtained:

$$
R_{0}=\rho\left(F V^{-1}\right)=\frac{\mu_{2} p \beta_{1}+\left[q+(1-p) \mu_{1}\right] \beta_{2}}{\mu_{2}\left(q+\mu_{1}\right)} \frac{\left\langle k^{2}\right\rangle}{\langle k\rangle} .
$$

Let $J_{0}$ be the Jacobian matrix of the middle three equations of model (2.1), then $J_{0}=F-V$ and $s\left(J_{0}\right)=\max \{\operatorname{Re} \lambda: \lambda$ is an eigenvalue of $\left.J_{0}\right\}, R_{0}<1 \Leftrightarrow s\left(J_{0}\right)<0, R_{0}>1 \Leftrightarrow s\left(J_{0}\right)>0$. [34]

Remark 3.1. Let $R_{0, a}=\frac{p \beta_{1}}{q+\mu_{1}} \frac{\left\langle k^{2}\right\rangle}{\langle k\rangle}, R_{0, d}=\frac{\left[q+(1-p) \mu_{1}\right] \beta_{2}}{\mu_{2}\left(q+\mu_{1}\right)} \frac{\left\langle k^{2}\right\rangle}{\langle k\rangle}$, then $R_{0}=R_{0, a}+R_{0, d}$, if $q=0$, this result is corresponding to the result in [27].

Remark 3.2. If we do not consider the asymptomatic infected state, that is $\beta_{1}=0, \mu_{1}=\mu_{2}=\mu, p=q=0$, then $R_{0}=\frac{\beta_{2}}{\mu} \frac{\left\langle k^{2}\right\rangle}{\langle k\rangle}$, which is corresponding to the result in $[6,33]$.

Lemma 3.2. If and only if $R_{0}>1$, there is a unique endemic equilibrium $E_{+}$.

Proof. We consider the right side of the model (2.1) to be equal to zero. Then, we obtain

$$
\left\{\begin{array}{l}
-\beta_{1} k S_{k} \theta_{1}-\beta_{2} k S_{k} \theta_{2}+\delta R_{k}=0 \\
\beta_{1} k S_{k} \theta_{1}+\beta_{2} k S_{k} \theta_{2}-\alpha E_{k}=0 \\
p \alpha E_{k}-q A_{k}-\mu_{1} A_{k}=0 \\
(1-p) \alpha E_{k}+q A_{k}-\mu_{2} I_{k}=0 \\
\mu_{1} A_{k}+\mu_{2} I_{k}-\delta R_{k}=0
\end{array}\right.
$$

Following the above equation, using $\theta=\beta_{1} \theta_{1}^{*}+\beta_{2} \theta_{2}^{*}$, we can find

$$
E_{k}^{*}=\frac{k \theta}{\alpha} S_{k}^{*}, A_{k}^{*}=\frac{p k \theta}{q+\mu_{1}} S_{k}^{*}, I_{k}^{*}=\frac{\left[q+(1-p) \mu_{1}\right] k \theta}{\mu_{2}\left(q+\mu_{1}\right)} S_{k}^{*}, R_{k}^{*}=\frac{k \theta}{\delta} S_{k}^{*} .
$$

According to the condition $N_{k}^{*}=S_{k}^{*}+E_{k}^{*}+A_{k}^{*}+I_{k}^{*}+R_{k}^{*}$, we can get

$$
S_{k}^{*}=\frac{\mu_{2} \alpha\left(q+\mu_{1}\right) \delta N_{k}^{*}}{\delta \mu_{2} \alpha\left(q+\mu_{1}\right)+\left\{\mu_{2} \alpha\left(q+\mu_{1}\right)+\delta \mu_{2}\left(q+\mu_{1}\right)+\delta \mu_{2} p \alpha\right.}
$$

Substituting $E_{k}^{*}, A_{k}^{*}$, and $I_{k}^{*}$ of Eq.(3.2) and Eq.(3.3) into $\theta$, we can obtain $\theta f(\theta)=0$, where

$$
f(\theta)=1-\frac{A_{1}}{\langle k\rangle} \sum_{k} \frac{k^{2} P_{k}}{A_{2}+A_{3} k \theta},
$$

and $\quad A_{1}=\delta \alpha\left\{\mu_{2} p \beta_{1}+\left[q+(1-p) \mu_{1}\right] \beta_{2}\right\}, \quad A_{2}=\delta \mu_{2}\left(q+\mu_{1}\right) \alpha$, $A_{3}=\mu_{2}\left(q+\mu_{1}\right) \alpha+\delta\left\{\mu_{2}\left(q+\mu_{1}\right)+\mu_{2} p \alpha+\alpha\left[q+(1-p) \mu_{1}\right]\right\}$. It is easy to find that $\theta f(\theta)=0$ has a trivial solution with no condition, and model (2.1) has a disease-free equilibrium. In addition,

$$
\begin{gathered}
\frac{d f(\theta)}{d \theta}=\frac{A_{1} A_{3}}{\langle k\rangle} \sum_{k} \frac{k^{3} P_{k}}{\left(A_{2}+A_{3} k \theta\right)^{2}}>0, \\
f\left(\beta_{1}+\beta_{2}\right)=1-\frac{A_{1}}{\langle k\rangle} \sum_{k} \frac{k^{2} P_{k}}{A_{2}+A_{3} k\left(\beta_{1}+\beta_{2}\right)} \\
>1-\frac{A_{1}}{\langle k\rangle} \sum_{k} \frac{k^{2} P(k)}{A_{3} k\left(\beta_{1}+\beta_{2}\right)}=1-\frac{A_{1}}{A_{3}\left(\beta_{1}+\beta_{2}\right)} \\
=1-\frac{\delta \alpha\left\{\mu_{2} p \beta_{1}+\left[q+(1-p) \mu_{1}\right] \beta_{2}\right\}}{\mu_{2}\left(q+\mu_{1}\right) \alpha+\delta\left\{\mu_{2}\left(q+\mu_{1}\right)+\mu_{2} p \alpha+\alpha\left[q+(1-p) \mu_{1}\right]\right\}} \frac{1}{\beta_{1}+\beta_{2}} \\
>1-\frac{\delta \alpha\left\{\mu_{2} p \beta_{1}+\left[q+(1-p) \mu_{1}\right] \beta_{2}\right\}}{\mu_{2}\left(q+\mu_{1}\right) \alpha+\delta\left\{\mu_{2}\left(q+\mu_{1}\right)+\mu_{2} p \alpha+\alpha\left[q+(1-p) \mu_{1}\right]\right\}}>0 .
\end{gathered}
$$

Thus, $\theta f(\theta)=0$ has a nontrivial solution if and only if $f(0)<0$, that is if and only if $R_{0}>1$, there is a unique endemic equilibrium.

\subsection{Stability of Disease-free and Endemic Equilibrium}

Theorem 3.1. If $R_{0} \leq 1, E_{0}$ of model (2.1) is locally asymptotically stable; if $R_{0}>1, E_{0}$ is unstable, and there is a unique endemic equilibrium $E_{+}$, and $E_{+}$is locally asymptotically stable.

Proof. From the results of Lemma 3.1, Lemma 3.2, and the basic reproduction number, we only need to prove the case of $R_{0}=1$. We consider the following model:

$$
\left\{\begin{array}{l}
\frac{d S_{k}}{d t}=-\beta_{1} k S_{k} \theta_{1}-\beta_{2} k S_{k} \theta_{2}+\delta R_{k}, \\
\frac{d E_{k}}{d t}=\beta_{1} k S_{k} \theta_{1}+\beta_{2} k S_{k} \theta_{2}-\alpha E_{k}, \\
\frac{d A_{k}}{d t}=p \alpha E_{k}-q A_{k}-\mu_{1} A_{k}, \\
\frac{d I_{k}}{d t}=(1-p) \alpha E_{k}+q A_{k}-\mu_{2} I_{k} .
\end{array}\right.
$$

The Jacobian matrix of model (3.4) at disease-free equilibrium is given by 


$$
J=\left(\begin{array}{cccc}
J_{11} & J_{12} & \cdots & J_{1 n} \\
J_{21} & J_{22} & \cdots & J_{2 n} \\
\vdots & \vdots & \ddots & \vdots \\
J_{n 1} & J_{n 2} & \cdots & J_{n n}
\end{array}\right)_{4 n \times 4 n}
$$

with

$$
J_{i j}=\left(\begin{array}{cccc}
-\delta & -\delta & -\frac{\beta_{1} P_{j} N_{i}}{\langle k\rangle N_{j}} i j-\delta & -\frac{\beta_{2} P_{j} N_{i}}{\langle k\rangle N_{j}} i j-\delta \\
0 & -\alpha & \frac{\beta_{1} P_{j} N_{i}}{\langle k\rangle N_{j}} i j & \frac{\beta_{2} P_{j} N_{i}}{\langle k\rangle N_{j}} i j \\
0 & p \alpha & -q-\mu_{1} & 0 \\
0 & (1-p) \alpha & q & -\mu_{2}
\end{array}\right) .
$$

The characteristic equation of the disease-free equilibrium is

$$
\begin{aligned}
& \left(\lambda+\mu_{2}\right)^{n-1}\left(\lambda+q+\mu_{1}\right)^{n-1}(\lambda+\delta)^{n}(\lambda+\alpha)^{n-1}\left(\lambda^{3}+H_{2} \lambda^{2}\right. \\
& \left.+H_{1} \lambda+H_{0}\right)=0
\end{aligned}
$$

where

$$
\begin{aligned}
& H_{2}=\alpha+q+\mu_{1}+\mu_{2}>0, \\
& H_{1}=\alpha\left(q+\mu_{1}\right)+\alpha \mu_{2}+\mu_{2}\left(q+\mu_{1}\right)-\left[p \alpha \beta_{1}+(1-p) \alpha \beta_{2}\right] \frac{\left\langle k^{2}\right\rangle}{\langle k\rangle}, \\
& H_{0}=\mu_{2} \alpha\left(q+\mu_{1}\right)-\alpha\left\{\mu_{2} p \beta_{1}+\left[q+(1-p) \mu_{1}\right] \beta_{2}\right\} \frac{\left\langle k^{2}\right\rangle}{\langle k\rangle},
\end{aligned}
$$

when $R_{0}=1, H_{0}=0$, and

$$
\begin{aligned}
& \alpha \mu_{2} p \beta_{1} \frac{\left\langle k^{2}\right\rangle}{\langle k\rangle}<\mu_{2} \alpha\left(q+\mu_{1}\right) \Rightarrow \alpha p \beta_{1} \frac{\left\langle k^{2}\right\rangle}{\langle k\rangle}<\alpha\left(q+\mu_{1}\right), \\
& (1-p) \alpha \mu_{1} \beta_{2} \frac{\left\langle k^{2}\right\rangle}{\langle k\rangle}<\mu_{2} \alpha\left(q+\mu_{1}\right) \Rightarrow(1-p) \alpha \beta_{2} \frac{\left\langle k^{2}\right\rangle}{\langle k\rangle}<\mu_{2} \\
& \left(\alpha+q+\mu_{1}\right) .
\end{aligned}
$$

Then, we obtain $H_{1}>0$, and the characteristic equation can be written as

$\lambda\left(\lambda+\mu_{2}\right)^{n-1}\left(\lambda+q+\mu_{1}\right)^{n-1}(\lambda+\delta)^{n}(\lambda+\alpha)^{n-1}\left(\lambda^{2}+H_{2} \lambda+H_{1}\right)=0$, where the eigenvalues of $J$ are all negative except zero eigenvalue when $R_{0}=1$.

\section{QUARANTINE STRATEGIES}

Quarantine is helpful in controlling diseases. In this section, we will discuss three different quarantine strategies for COVID-19 disease model on networks.

\subsection{Proportional Quarantine}

In this case, for fixed spreading rates $\beta_{1}$ and $\beta_{2}$, let $\omega(0<\omega<1)$ is the density of quarantine nodes in the network. At the mean-field level, the presence of proportional quarantine will effectively reduce the spreading rate. Thus, we can approximately use $\beta_{1}(1-\omega)$ to substitute $\beta_{1}$ and use $\beta_{2}(1-\omega)$ to substitute $\beta_{2}$; thus, the system (2.1) becomes

$$
\left\{\begin{array}{l}
\frac{d S_{k}(t)}{d t}=-\beta_{1}(1-\omega) k S_{k}(t) \theta_{1}(t)-\beta_{2}(1-\omega) k S_{k}(t) \theta_{2}(t)+\delta R_{k}(t) \\
\frac{d E_{k}(t)}{d t}=\beta_{1}(1-\omega) k S_{k}(t) \theta_{1}(t)+\beta_{2}(1-\omega) k S_{k}(t) \theta_{2}(t)-\alpha E_{k}(t) \\
\frac{d A_{k}(t)}{d t}=p \alpha E_{k}(t)-q A_{k}(t)-\mu_{1} A_{k}(t) \\
\frac{d I_{k}(t)}{d t}=(1-p) \alpha E_{k}(t)+q I_{k}(t)-\mu_{2} I_{k}(t) \\
\frac{d R_{k}(t)}{d t}=\mu_{1} A_{k}(t)+\mu_{2} I_{k}(t)-\delta R_{k}(t)
\end{array}\right.
$$

Using the same method in Section 3, we obtain a selfconsistency equation as follows:

$$
\theta=\frac{\delta \alpha(1-\omega) A_{1}}{\langle k\rangle} \sum_{k} \frac{\left\langle k^{2}\right\rangle P_{k}}{A_{2}+A_{3} k \theta} \theta \equiv \tilde{f}(\theta) .
$$

By arguments similar to those in Section 3, the epidemic threshold $\widetilde{R_{w}}$ is determined by the following inequality:

$$
\left.\frac{d \tilde{f}(\theta)}{d \theta}\right|_{\theta=0}>1
$$

therefore, it can be shown that

$$
\widetilde{R_{w}}=\frac{\mu_{2} p(1-\omega) \beta_{1}+\left[q+(1-p) \mu_{1}\right](1-\omega) \beta_{2}}{\mu_{2}\left(q+\mu_{1}\right)} \frac{\left\langle k^{2}\right\rangle}{\langle k\rangle},
$$

that is,

$$
\widetilde{R_{w}}=(1-\omega) R_{0}
$$

Note that in (4.3), when $\omega=0$, that is, if no quarantine were done, then $\widetilde{R_{w}}=R_{0}$; when $0<\omega<1, \widetilde{R_{w}}<R_{0}$, that is, the quarantine scheme is effective; while as $\omega \rightarrow 1, \widetilde{R_{w}} \rightarrow 0$, that is, in the case of a full quarantine, it would be impossible for the epidemic to spread in the network.

\subsection{Targeted Quarantine}

While proportional quarantine schemes are effective, there may be more efficient schemes due to the heterogeneous nature of scale-free networks. We introduce an upper threshold $\kappa$ such that all nodes with connectivity $k>\kappa$ are immunized, that is, we define the quarantine rate $\omega_{k}$ by

$$
\omega_{k}= \begin{cases}1, & k>\kappa \\ c, & k=\kappa \\ 0, & k<\kappa\end{cases}
$$

where $0<c<1$ and $\sum_{k} \omega_{k} P(k)=\bar{\omega}$, where $\bar{\omega}$ is the average quarantine rate. The epidemic dynamics model is 

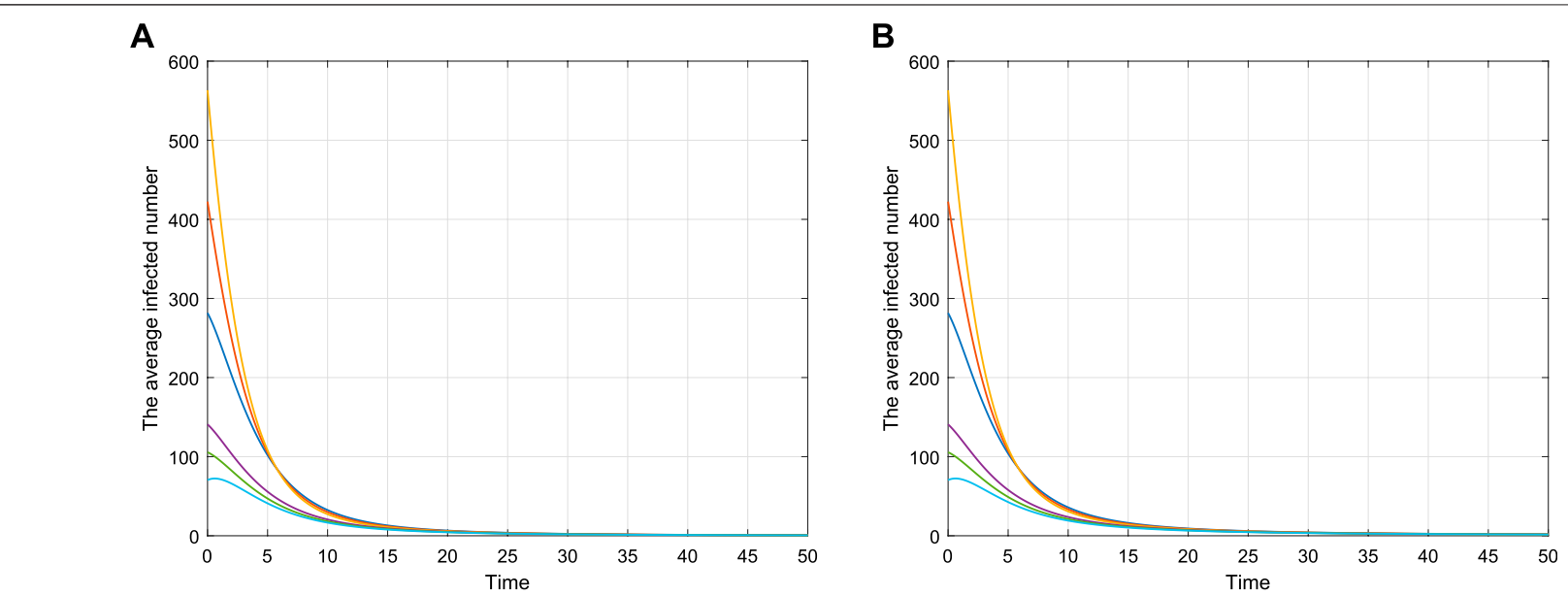

FIGURE 2 | Asymptotical stability of the model.

\begin{tabular}{|c|c|c|c|c|c|c|c|c|c|}
\hline Figure 2 & $\beta_{1}$ & $\beta_{2}$ & $\delta$ & $a$ & $p$ & $\mathbf{q}$ & $\mu_{1}$ & $\mu_{2}$ & $\boldsymbol{R}_{0}$ \\
\hline$A$ & 0.08 & 0.08 & 0.4 & 0.4 & 0.5 & 0.5 & 0.2 & 0.4 & $0.8193<1$ \\
\hline$B$ & 0.0905 & 0.10003 & 0.4 & 0.4 & 0.5 & 0.5 & 0.2 & 0.4 & 1 \\
\hline
\end{tabular}

TABLE 2 | Parameters of Figure 4

\begin{tabular}{|c|c|c|c|c|}
\hline & $\beta_{1}$ & $\beta_{2}$ & $\Delta$ & $\alpha$ \\
\hline \multirow[t]{2}{*}{ Figure 4} & 0.0020178 & 0.033949 & 0.12178 & 0.1055744 \\
\hline & $p$ & $q$ & $\mu_{1}$ & $\mu_{2}$ \\
\hline Figure 4 & 0.40043 & 0.040403 & $0.0119,536$ & 0.20955 \\
\hline
\end{tabular}

$$
\left\{\begin{array}{l}
\frac{d S_{k}(t)}{d t}=-\beta_{1}\left(1-\omega_{k}\right) k S_{k}(t) \theta_{1}(t)-\beta_{2}\left(1-\omega_{k}\right) k S_{k}(t) \theta_{2}(t)+\delta R_{k}(t) \\
\frac{d E_{k}(t)}{d t}=\beta_{1}\left(1-\omega_{k}\right) k S_{k}(t) \theta_{1}(t)+\beta_{2}\left(1-\omega_{k}\right) k S_{k}(t) \theta_{2}(t)-\alpha E_{k}(t) \\
\frac{d A_{k}(t)}{d t}=p \alpha E_{k}(t)-q A_{k}(t)-\mu_{1} A_{k}(t) \\
\frac{d I_{k}(t)}{d t}=(1-p) \alpha E_{k}(t)+q I_{k}(t)-\mu_{2} I_{k}(t) \\
\frac{d R_{k}(t)}{d t}=\mu_{1} A_{k}(t)+\mu_{2} I_{k}(t)-\delta R_{k}(t)
\end{array} .\right.
$$

This leads to

$$
\theta=\frac{\delta \alpha A_{1}}{\langle k\rangle} \sum_{k} \frac{\left\langle k^{2}\right\rangle P_{k}\left(1-\omega_{k}\right)}{A_{2}+A_{3} k \theta} \theta \equiv \tilde{f}(\theta) .
$$

Therefore, the epidemic threshold

$$
\widehat{R_{w}}=\frac{\mu_{2} p \beta_{1}+\left[q+(1-p) \mu_{1}\right] \beta_{2}}{\mu_{2}\left(q+\mu_{1}\right)} \frac{\left\langle k^{2}\right\rangle-\left\langle k^{2} \omega_{k}\right\rangle}{\langle k\rangle} .
$$

Note that $\left\langle k^{2} \omega_{k}\right\rangle=\bar{\omega}\left\langle k^{2}\right\rangle+\sigma^{\prime}$, where $\sigma^{\prime}=\left\langle\left(\omega_{k}-\bar{\omega}\right) \times\left[k^{2}-\right.\right.$ $\left.\left.\left\langle k^{2}\right\rangle\right]\right\rangle$ is the covariance of $\omega_{k}$ and $k^{2}$. There may be $\kappa$ (usually big enough) where $\sigma^{\prime}<0$, but for appropriately small $\kappa, \omega_{k}-\bar{\omega}$ and $k^{2}-\left\langle k^{2}\right\rangle$ have the same signs except for some $k$ where $\omega_{k}-$ $\bar{\omega}$ and/or $k^{2}-\left\langle k^{2}\right\rangle$ is zero; Therefore $\sigma^{\prime}>0$ for appropriate $\kappa$. Then,

$$
\widehat{R_{w}}<\frac{1-\bar{\omega}}{1-\omega} \widetilde{R_{w}} .
$$

If we set $\bar{\omega}=\omega$, then,

$$
\widehat{R_{w}}<\widetilde{R_{w}}(0<\omega<1),
$$

which means the targeted quarantine scheme is more efficient than the uniform quarantine scheme for the same average quarantine rate.

\subsection{High-Risk Quarantine}

If a neighbor of a susceptible individual is infected, vaccinating this susceptible individual is 

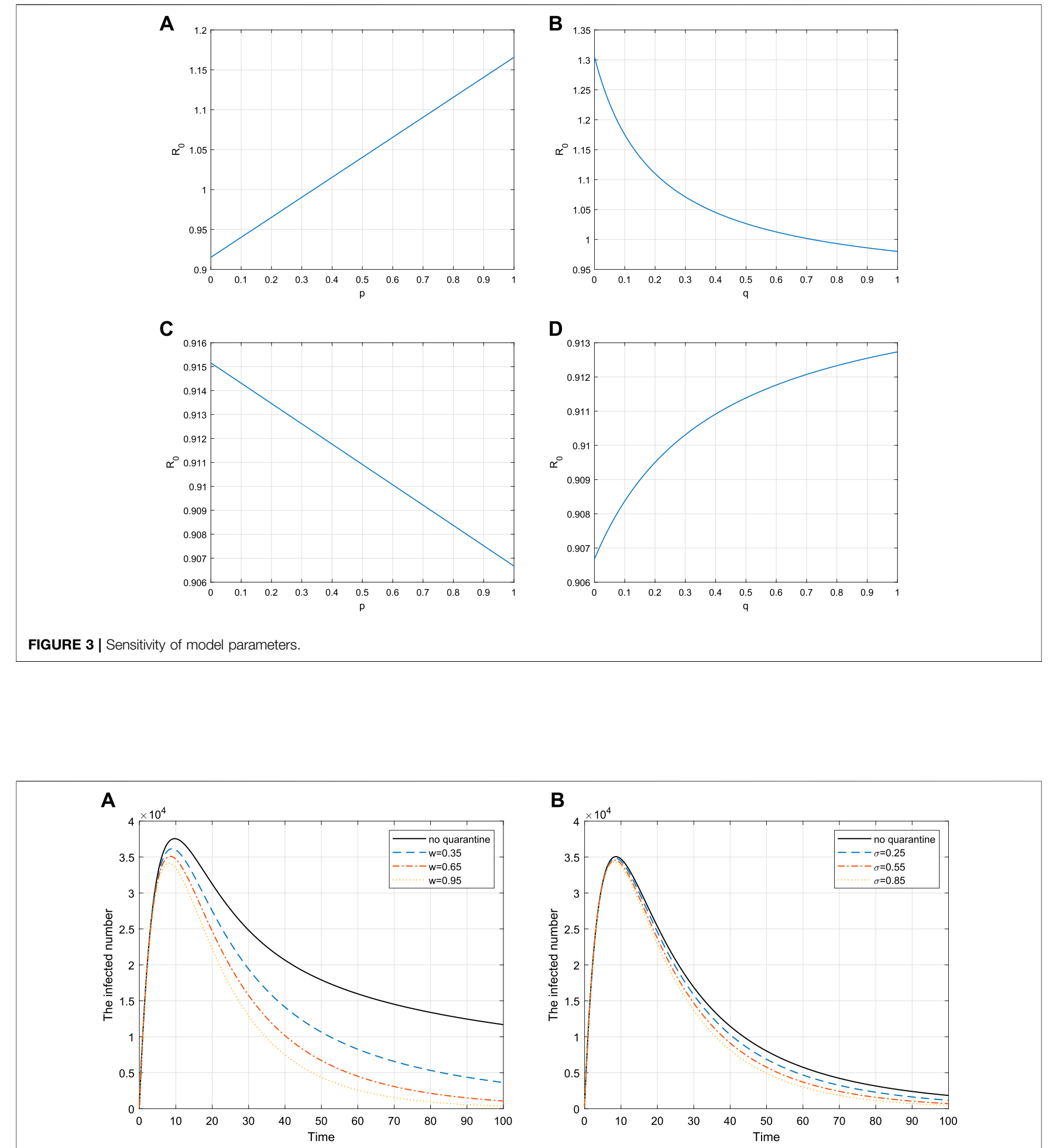

FIGURE 4 | Effectiveness of quarantine for different $\omega$ and $\sigma$ respectively.

called high-risk immunity [37]. We introduce the parameter $\sigma$, that is, the probability that a node is quarantined per unit time, and $\Gamma=\frac{k P_{k}}{\langle k\rangle N}$, which denotes the probability that any given node is the neighbor of some specific nodes. The epidemic dynamics model is 


$$
\left\{\begin{array}{l}
\frac{d S_{k}(t)}{d t}=-\beta_{1}(1-\sigma \Gamma) k S_{k}(t) \theta_{1}(t)-\beta_{2}(1-\sigma \Gamma) k S_{k}(t) \theta_{2}(t)+\delta R_{k}(t) \\
\frac{d E_{k}(t)}{d t}=\beta_{1}(1-\sigma \Gamma) k S_{k}(t) \theta_{1}(t)+\beta_{2}(1-\sigma \Gamma) k S_{k}(t) \theta_{2}(t)-\alpha E_{k}(t) \\
\frac{d A_{k}(t)}{d t}=p \alpha E_{k}(t)-q A_{k}(t)-\mu_{1} A_{k}(t) \\
\frac{d I_{k}(t)}{d t}=(1-p) \alpha E_{k}(t)+q I_{k}(t)-\mu_{2} I_{k}(t) \\
\frac{d R_{k}(t)}{d t}=\mu_{1} A_{k}(t)+\mu_{2} I_{k}(t)-\delta R_{k}(t)
\end{array}\right.
$$

This leads to

$$
\theta=\frac{\delta \alpha A_{1}}{\langle k\rangle} \sum_{k} \frac{\left\langle k^{2}\right\rangle P_{k}(1-\sigma \Gamma)}{A_{2}+A_{3} k \theta} \theta \equiv \bar{f}(\theta) .
$$

And the epidemic threshold

$$
\bar{R}_{0}=\frac{\mu_{2} p \beta_{1}+\left[q+(1-p) \mu_{1}\right] \beta_{2}}{\mu_{2}\left(q+\mu_{1}\right)}\left(\frac{\left\langle k^{2}\right\rangle}{\langle k\rangle}-\frac{\sigma\left\langle k^{3} P_{k}\right\rangle}{\langle k\rangle^{2} N}\right)<R_{0} .
$$

This means that the implementation of high-risk quarantine is effective, and the longer the period of $\sigma\left\langle k^{3} P_{k}\right\rangle$, the more effective it is.

\section{NUMERICAL SIMULATIONS}

In this section, we present numerical simulations of model (2.1), (4.1) and (4.6) to illustrate the results in Section 3 and 4. Without loss of generality, we consider the dynamical process on scale-free networks with $P_{k}=c k^{-\gamma}$ and $\sum_{k=1}^{n} P_{k}=1$, $\gamma=3, n=200$. The average infected number is $A(t)+I(t)=\sum_{k=1}^{n} A_{k}(t) P_{k}+\sum_{k=1}^{n} I_{k}(t) P_{k}$. The parameters of the model are estimated by MCMC using the number of cases in Wuhan (http://wjw.wuhan.gov.cn/ztzl_28/fk/yqtb/).

Figure 2 shows the asymptotical stability of model (2.1) with different values, and the parameter values are shown in Table $\mathbf{1 .}$ Figures 2A,B show the time series of the average infected number $I(t)$ with different initial values, which is in accord with the asymptotical stability of the disease-free equilibrium.

The sensitivity analysis of the basic reproduction number $R_{0}$ can be performed by model parameters. It is easy to obtain that

$$
\begin{gathered}
\frac{\partial R_{0}}{\partial \beta_{1}}=\frac{p}{q+\mu_{1}} \frac{\left\langle k^{2}\right\rangle}{\langle k\rangle}>0, \quad \frac{\partial R_{0}}{\partial \beta_{2}}=\frac{q+(1-p) \mu_{1}}{\left(q+\mu_{1}\right) \mu_{2}} \frac{\left\langle k^{2}\right\rangle}{\langle k\rangle}>0, \\
\frac{\partial R_{0}}{\partial \mu_{1}}=\frac{-\beta_{2} p q-\beta_{1} \mu_{2} p}{\mu_{2}\left(q+\mu_{1}\right)^{2}} \frac{\left\langle k^{2}\right\rangle}{\langle k\rangle}<0, \quad \frac{\partial R_{0}}{\partial \mu_{2}}=-\frac{\beta_{2}\left[q+(1-p) \mu_{1}\right]}{\mu_{2}^{2}\left(q+\mu_{1}\right)} \frac{\left\langle k^{2}\right\rangle}{\langle k\rangle}<0, \\
\frac{\partial R_{0}}{\partial p}=\frac{\beta_{1} \mu_{2}-\beta_{2} \mu_{1}}{\mu_{2}\left(q+\mu_{1}\right)} \frac{\left\langle k^{2}\right\rangle}{\langle k\rangle}, \quad \frac{\partial R_{0}}{\partial q}=\frac{p\left(\beta_{2} \mu_{1}-\beta_{1} \mu_{2}\right)}{\mu_{2}\left(q+\mu_{1}\right)^{2}} \frac{\left\langle k^{2}\right\rangle}{\langle k\rangle} .
\end{gathered}
$$

Equation (5.1) shows $R_{0}$ is linearly positively correlated with $\beta_{1}$ and $\beta_{2} ; R_{0}$ is negatively correlated with $\mu_{1}$ and $\mu_{2}$; the correlation between $R_{0}$ and $p$ or $q$ is determined by $\beta_{2} \mu_{1}-\beta_{1} \mu_{2}$. As can be seen from Figure 3 that the monotonicity of R0 increases or decreases with respect to $\mathrm{p}$ and $\mathrm{q}$, respectively. In Figure 4, the parameters of the model are estimated by MCMC using the number of cases in
Wuhan and the parameter values are shown in Table 2. Figure 4 shows the change of total infection density with time under different $\omega$ and $\sigma$. It can be seen that with the increase of $\omega$ and $\sigma$, the total infection density is becoming lower as $\omega$ and $\sigma$ increase.

\section{CONCLUSION AND DISCUSSION}

In this article, we have generalized a traditional homogeneous epidemic model with asymptomatic infectors to a network case to study the spread of COVID-19. As one can see, homogeneous epidemic models can be considered as a special case of network epidemic models, and network epidemic models are more realistic and refined description of disease propagation in population. Considering two cases of infected state (show symptoms and show no symptoms), we investigated an SEAIRS model of COVID-19 on scale-free networks to approach the disease progress and calculated the basic reproduction number $R_{0}$. By theoretical analysis, we obtained the asymptotical stability of the equilibria: if $R_{0} \leq 1, E_{0}$ is asymptotically stable. Then, we analyzed the different quarantine strategies in the model. At last, numerical simulations illustrated the theoretical analysis. The results show that the asymptomatic infectors may infect health individuals as well as symptomatic infectors.

The SEAIRS model is an extension of the SEAIRS model, and it is a case of the $S \rightarrow S$ model. The $S \rightarrow S$ model, $S \rightarrow I$ model, and $S \rightarrow R$ model differ in terms of pathological mechanisms. It is difficult to say which one is better than others except introducing a special disease. Within $S \rightarrow S$ models, formally, either the SIS model or SIRS model can be a special case of the SEIRS model. The parameters $\beta_{1}$ and $\beta_{2}$ denoted in the model are constant. In fact, many infectious diseases show seasonal phenomenon, such as measles, chickenpox, and mumps [36], and each individual has a periodic order; the parameters above should have some periodic property.

At the same time, we have also discussed proportional, targeted quarantine and high-risk quarantine schemes for network models. The result of Leung et al. [27] by illustration is suitable for Norovirus and measles. Different from their work, the formulaic result seems to be unsuitable for any kind of disease, for none of the parameters in the model is assigned to a specific value. In fact, for different disease, the parameters in the model may present special values. COVID-19 is now spreading faster abroad. Susceptible people will also be infected by asymptomatic patients. If not controlled, the epidemic will spread more widely and the number of infected people will be more difficult to control. The epidemic situation in China was obviously well controlled after the implementation of the strategy of lockdown, and other quarantine strategies. Therefore, the model and quarantine strategy in this article can also be applied to other countries. And, we hope that the results will be helpful to study and control the spread of COVID-19 disease.

\section{DATA AVAILABILITY STATEMENT}

The original contributions presented in the study are included in the article/Supplementary Material; further inquiries can be directed to the corresponding author. 


\section{AUTHOR CONTRIBUTIONS}

ML and YS designed the study, and WL and ML carried out the analysis and contributed to writing the article. XF and YS performed numerical simulations.

\section{REFERENCES}

1. Report on novel coronavirus pneumonia epidemic in real time. (2020) https:// voice.baidu.com/act/newpneumonia/newpneumonia/.

2. Tang B, Wang X, Li Q, Bragazzi NL, Tang SY, Xiao YN et al, Estimation of the transmission risk of the $2019-\mathrm{nCoV}$ and its implication for public health interventions. J Comput Math (2020) 9:462. doi:10.3390/jcm9020462

3. Tang B, Bragazzi NL, Li Q, Tang S, Xiao Y and Wu J. An updated estimation of the risk of transmission of the novel coronavirus (2019-nCov). Infect Dis Model (2020) 5:248-55. doi:10.1016/j.idm.2020.02.001

4. Hethcote HW. Three basic epidemiological models. Appl Math Ecol (1989) 355: 119-44.

5. Li MY, Muldowney JS and van den Driessche P. Global stability of SEIRS models in epidemiology. Can Appl Math Q (1999) 7:409-25.

6. Pastor-Satorras R and Vespignani A. Epidemic spreading in scale-free networks. Phys Rev Lett (2001) 86:3200-3. doi:10.1103/PhysRevLett.86.3200

7. Butts CT. Revisiting the foundations of network analysis. Science (2009) 325(5939):414-6. doi:10.1126/science.1171022

8. Newman M. Networks: an introduction. New York, NY, USA: Oxford University Press (2010).

9. Vespignani A. Predicting the behavior of techno-social systems. Science (2009) 325(5939):425-8. doi:10.1126/science.1171990

10. Vespignani A. Modeling dynamical processes in complex socio-technical systems. Nat Phys (2012) 8:32-9. doi:10.1038/nphys 2160

11. Pastor-Satorras R, Castellano C, Van Mieghem P and Vespignani A. Epidemic processes in complex networks. Rev Mod Phys (2015) 87:925-79. doi:10.1017/ cbo9780511791383.010

12. Wang B, Han Y and Tanaka G. Interplay between epidemic spread and information propagation on metapopulation networks. J Theor Biol (2017) 420:18-25. doi:10.1016/j.jtbi.2017.02.020

13. Liu G, Liu Z and Jin Z. Dynamics analysis of epidemic and information spreading in overlay networks. J Theor Biol (2018) 444:28-37. doi:10.1016/j. jtbi.2018.02.010

14. Zhang $X$, Shan C, Jin Z and Zhu H. Complex dynamics of epidemic models on adaptive networks. J Differ Equ (2019) 266(1):803-32. doi:10.1016/j.jde.2018. 07.054

15. Massad E, Ma S, Chen M, Struchiner C, Stollenwerk N and Mara A. Scale-free network of a dengue epidemic. Appl Math Comput (2008) 195:376-81. doi:10. 1016/j.amc.2007.04.102

16. Mishra BK and Pandey SK. Dynamic model of worms with vertical transmission in computer network. Appl Math Comput (2011) 217: 8438-46. doi:10.1016/j.amc.2011.03.041

17. Zhang JP and Jin Z. The analysis of an epidemic model on networks. Appl Math Comput (2011) 217:7053-64. doi:10.1007/978-3-662-47824-0_3

18. Nian F and Yao S. The epidemic spreading on the multi-relationships network. Appl Math Comput (2018) 339:866-73. doi:10.1016/j.amc.2018.07.030

19. Wang ZS, Guo QT, Sun SW and Xia CY. The impact of awareness diffusion on SIR-like epidemics in multiplex networks. Appl Math Comput (2019) 349: 134-47. doi:10.1016/j.chaos.2019.109425

20. Wang ZS, Xia CY, Chen ZQ and Chen GR. Epidemic propagation with positive and negative preventive information in multiplex networks. IEEE Trans Cybern (2020) doi:10.1109/TCYB.2019.2960605

21. Grunnill M. An exploration of the role of asymptomatic infections in the epidemiology of dengue viruses through susceptible, asymptomatic, infected and recovered (SAIR) models. J Theor Biol (2018) 439:195-204. doi:10.1016/j. jtbi.2017.12.009

\section{FUNDING}

Research Project Supported by Fund for Shanxi 1331KIRT and the outstanding youth fund of North University of China.

22. Mishra A and Gakkhar S. Modeling of Dengue with impact of asymptomatic infection and ADE factor. Differ Equ Dyn Syst (2018) 19:1-17. doi:10.1007/s12591018-0436-4

23. Beretta E, Capasso V and Garao DG. A mathematical model for malaria transmission with asymptomatic carriers and two age groups in the human population. Math Biosci (2018) 300:87-101. doi:10.1016/j.mbs.2018.03.024

24. Gallimore CI, Cubitt D, du Plessis N and Gray JJ. Asymptomatic and symptomatic excretion of noroviruses during a hospital outbreak of gastroenteritis. J Clin Microbiol (2004) 42:2271-4. doi:10.1128/jcm.42.5.2271-2274.2004

25. García C, DuPont HL, Long KZ, Santos JI and Ko G. Asymptomatic norovirus infection in Mexican children. J Clin Microbiol (2006) 44:2997-3000. doi:10. 1128/JCM.00065-06

26. Bucardo F, Nordgren J, Carlsson B, Kindberg E, Paniagua M, Möllby R, et al, and Svensson L. Asymptomatic norovirus infections in Nicaraguan children and its association with viral properties and histo-blood group antigens. Pediatr Infect Dis J (2010) 29:934-9. doi:10.1097/INF.0b013e3181ed9f2f

27. Leung KY, Trapman $P$ and Britton T. Who is the infector? Epidemic models with symptomatic and asymptomatic cases. Math Biosci (2018) 301:190-8. doi:10.1016/j.mbs.2018.04.002

28. Chisholm RH, Campbell PT, Wu Y, Tong SYC, McVernon J and Geard N Implications of asymptomatic carriers for infectious disease transmission and control. $R$ Soc Open Sci (2018) 5:172341. doi:10.1098/rsos.172341

29. De la Sen M, Alonso-Quesada S, Ibeas A and Nistal R. On an SEIADR epidemic model with vaccination, treatment and dead-infectious copses removal controls. Math Comput Simul (2019) 163:47-79. doi:10.3390/ijerph16152689

30. Ma Y, Liu M, Hou Q and Zhao J. Modelling seasonal HFMD with the recessive infection in Shandong, China. Math Biosci Eng (2013) 10:1159-71. doi:10. 3934/mbe.2013.10.1159

31. Cao J, Wang Y, Alofi A, Mazrooei AA and Elaiw A. Global stability of an epidemic model with carrier state in heterogeneous networks. IMA J Appl Math (2015) 80:1025. doi:10.1093/imamat/hxu040

32. van den Driessche $\mathrm{P}$ and Watmough J. Reproduction numbers and sub-threshold endemic equilibria for compartmental models of disease transmission. Math Biosci (2002) 180:29-48. doi:10.1016/s0025-5564(02)00108-6

33. Liu J and Zhang T. Epidemic spreading of an SEIRS model in scale-free networks. Commun Nonlinear Sci Numer Simulat (2011) 16:3375-84. doi:10.1063/1.5001176

34. Zhao XQ and Jing ZJ. Global asymptotic behavior in some cooperative systems of functional differential equations. Can Appl Math Q (1996) 4:421-44.

35. Barthélemy M, Barrat A, Pastor-Satorras R and Vespignani A. Velocity and hierarchical spread of epidemic outbreaks in scale-free networks. Phys Rev Lett (2004) 92:178701. doi:10.1103/PhysRevLett.92.178701

36. Yorke JA and London WP. Recurrent outbreaks of measles, chickenpox and mumps. II. Systematic differences in contact rates and stochastic effects. Am J Epidemiol (1973) 98:469-82. doi:10.1093/oxfordjournals.aje.a121575

37. Nian $\mathrm{F}$ and Wang X. Efficient immunization strategies on complex networks. J Theor Biol (2010) 264:77-83. doi:10.1016/j.jtbi.2010.01.007

Conflict of Interest: The authors declare that the research was conducted in the absence of any commercial or financial relationships that could be construed as a potential conflict of interest.

Copyright (c) $2021 \mathrm{Li}, \mathrm{Fu}$, Sun and Liu. This is an open-access article distributed under the terms of the Creative Commons Attribution License (CC BY). The use, distribution or reproduction in other forums is permitted, provided the original author(s) and the copyright owner(s) are credited and that the original publication in this journal is cited, in accordance with accepted academic practice. No use, distribution or reproduction is permitted which does not comply with these terms. 